Check for updates

Cite this: Mater. Adv., 2022, 3, 2505

Received 10th December 2021, Accepted 13th January 2022

DOI: 10.1039/d1ma01169c

rsc.li/materials-advances

\title{
Vaccine adjuvant platform and fluorescence imaging of amphiphilic $\gamma$-PGA-IMQ-LA-FL conjugates $\dagger$
}

\author{
Wenzhu Yin, (D) ab Dechun Xuan, ${ }^{c}$ Bihua Deng, ${ }^{a}$ Mingxu Zhou, (D) a Fang Ma, ${ }^{a}$ \\ Jinqiu Zhang ${ }^{\star a c}$ and Yu Lu*abc
}

\begin{abstract}
Adjuvants have proven to be integral components in most vaccines for promoting appropriate immune responses at both innate and adaptive levels. Imiquimod (IMQ, R837), an agonist for Toll-like receptors 7 (TLR7), is a potent molecular activator of the innate immune system and has been developed as an adjuvant for immunotherapy. However, due to its pharmacokinetic profile, IMQ may induce strong local and systemic inflammatory reactions and is poorly tolerated. To overcome these hurdles, herein, we conjugate IMQ and lauryl alcohol (LA) to the backbone of biodegradable poly ( $\gamma$-glutamic acid) $(\gamma$-PGA) to prepare an amphiphilic polymer (shortened to $\gamma$-PGA-IMQ-LA) with improved stability and biodistribution of IMQ. In order to investigate the distribution of the amphiphilic polymer in vitro and in vivo, fluorescein was grafted to the lateral chain to synthesize fluorescent-labeled $\gamma-P G A-I M Q-L A-F L$ (shortened to FIP). The novel amphiphilic FIP polymer was characterized via ${ }^{1} \mathrm{H} N \mathrm{NR}, \mathrm{FI}-\mathrm{IR}, \mathrm{UV}$-vis, fluorescence spectra and zeta potential. Using ovalbumin (OVA) as a model antigen and FIP as an adjuvant for immunization, the formulations of FIP + OVA in different dosages could significantly promote the production of OVA-specific lgG in mice and prolong immune responses with acceptable safety.
\end{abstract}

\section{Introduction}

Among the various health interventions, vaccination is undoubtedly one of the most successful methods and continues to have a huge impact on the prevention of deaths caused by global infectious diseases. ${ }^{1}$ Since the first introduction of the smallpox vaccine by Edward Jenner in 1798, hundreds of vaccines have been developed to protect against a range of infectious diseases. ${ }^{2}$ Although traditional vaccines, including inactivated pathogens or attenuated variants, can induce potent immune responses and provide protection against infections, safety concerns have limited their clinical application, especially in individuals with compromised immune systems. ${ }^{3,4}$ To solve these problems, subunit and recombinant vaccines generated from modern biotechnology have been explored because of their higher

\footnotetext{
${ }^{a}$ Institute of Veterinary Immunology \& Engineering, National Research Center of Engineering and Technology for Veterinary Biologicals, Jiangsu Key Laboratory for Food Quality and Safety-State Key Laboratory Cultivation Base, Ministry of Science and Technology, Jiangsu Academy of Agricultural Sciences, Nanjing 210014, China

${ }^{b}$ Jiangsu Co-innovation Center for Prevention and Control of Important Animal Infectious and Zoonoses, Yangzhou, 225009, China

'School of Pharmacy, Jiangsu University, Zhenjiang 212013, China

† Electronic supplementary information (ESI) available. See DOI: 10.1039/ d1ma01169c
}

purities and better tolerance compared with inactivated or liveattenuated pathogens. Unfortunately, these new vaccines have insufficient immunogenicity and do not induce effective immune responses when used alone and, therefore, must be delivered with immunostimulatory adjuvants to enhance the vaccine potency. ${ }^{5,6}$

Adjuvants are usually considered to be integral components that are capable of enhancing and/or shaping antigen-specific immune responses effectively ${ }^{7}$ and reducing antigen dosage. Adjuvants in development, or in use currently, include aluminum salts, oil emulsions, saponins, immune stimulating complexes, liposomes, microparticles, nonionic block copolymers, polysaccharides, cytokines and bacterial derivatives. ${ }^{8}$ However, many adjuvants may cause headaches, inflammation and other adverse effect, which restricts their uses in vaccines. An ideal vaccine adjuvant should be compatible with antigens, biologically safe, capable of eliciting the expected immune responses, easy to use, easily prepared and inexpensive. With the growing understanding of the important role of adjuvants in vaccines, there is an urgent need to develop novel adjuvants that can enhance and shape vaccine-elicited responses. ${ }^{9}$

Toll-like receptor (TLR) agonists are key to the successful design of potent vaccines and cancer immunotherapeutics. TLR agonists are a class of transmembrane pattern recognition receptors (PRRs) and are widely distributed amongst innate immune cell subsets over a broad range of species. ${ }^{10,11}$ TLR 7 
agonists, such as imiquimod (IMQ, R837), have been examined as vaccine adjuvants in a number of clinical trials. ${ }^{12-15}$ However, their pharmacokinetic profiles are characterized by rapid systemic dissemination upon local (e.g. subcutaneous or intramuscular) administration, thereby causing unwanted innate immune activation at multiple distal tissues. ${ }^{16}$ In addition, they are water-insoluble drugs and exhibit unacceptable systemic toxicity, which is currently a strong limitation in applying IMQ in mass immunization campaigns. In the context of vaccination, the rapid diffusion of molecular adjuvants dramatically lowers the ability of antigen and TLR agonists to reach the same antigen-presenting cells in the draining lymph node (DLN), which results in suboptimal immunity to the delivered antigen and in consequence wasted inflammation. ${ }^{17}$ To mitigate these limitations, it has become an attractive target for us to utilize covalent ligation of TLR agonists to polymeric nanoparticles, which might provide chemically better-defined alternatives to physical (co)encapsulation systems to improve its biodistribution and pharmacokinetics. ${ }^{18-20}$

Poly $(\gamma$-glutamic acid) ( $\gamma$-PGA) is a water soluble anionic bioderived polypeptide polymer that has been used in a diverse range of applications including in the food industry, cosmetics, agriculture, carrier and drug delivery. ${ }^{21,22}$ Biodegradable nanoparticles (NPs) composed of $\gamma$-PGA with L-phenylalanine ethyl ester have been shown to generate antigen-specific cellular and humoral immune responses after immunization with various antigens. ${ }^{23,24}$ The structure of liposomes allows them to interact with various cells and components of the immune system, rendering them ideal candidates for antigen delivery. ${ }^{25-27}$ The conjugation of a small molecule TLR7 agonist to macromolecular constructs provides the possibility to impede systemic clearance, positively affecting the pharmacokinetic profiles. ${ }^{28,29}$ In addition, lipid amphiphiles exhibit an affinity to albumin and lipoproteins and hence promote lymphatic translocation to lymphoid tissues by "hitching a ride" along the interstitial albumin flow. ${ }^{15}$ Although adjuvant effects of TLR7/8 agonists and water-soluble $\gamma$-PGA derivatives are well-documented, respectively, only limited studies have attempted to incorporate these TLR7/8 agonists into water-soluble $\gamma$-PGA derivative nanoparticles as adjuvants. ${ }^{30-33}$

In the present work, we firstly grafted IMQ to the $\gamma$-PGA skeleton through covalent bonding to improve its lymphatic delivery and pharmacokinetics. Furthermore, we designed an amphiphilic covalently bound fluoresceinamine, IMQ and lauryl alcohol (LA) to $\gamma$-PGA to generate an amphiphilic polymer $\gamma$-PGA-IMQ-LA with improved stability and biodistribution of IMQ. It is well known that water-soluble drugs find it difficult to penetrate the cell membrane to get into the cell. So it requires the modification of hydrophobic groups, such as alkyl chains, to increase its lipophilicity for membrane permeability. At the same time, the amphiphile molecules can self-assemble into nanostructures such as fibrils, micelles, and other structures for vaccine adjuvants. ${ }^{34}$ To assess the cell uptake of the amphiphilic polymer $\gamma$-PGA-IMQ-LA, we grafted fluorescein to the lateral chain to synthesise fluorescent labeling $\gamma$-PGA-IMQLA-FL (shortened to FIP) (Scheme 1). The novel amphiphilic FIP

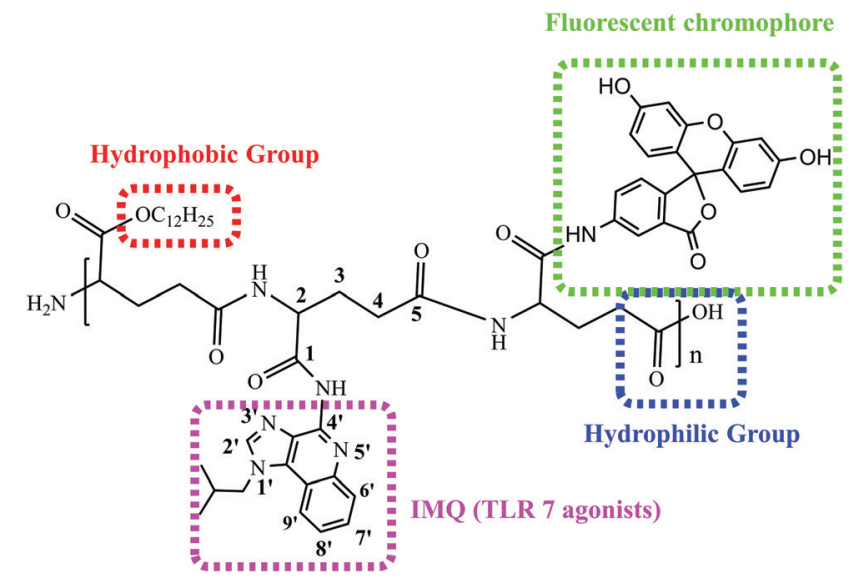

Scheme 1 The structure of the amphiphilic polymer FIP.

polymer was characterized using ${ }^{1} \mathrm{H}$ NMR, UV-vis, fluorescence, FI-IR and zeta potential. Using ovalbumin (OVA) as a model antigen and FIP as an adjuvant in mice for immunization, the formulation of FIP + OVA could enhance the production of OVAspecific IgG and promote immune responses with acceptable safety. In contrast to pure lipidation, the conjugated amphiphilic polymer is very water-soluble which is beneficial for injection. The amphiphilic FIP conjugates showed antigen-specific humoral and cellular immune response synergistic effects. Moreover, the FIP adjuvant could be prepared into different formulations for commercialization and used for fluorescence tracing.

\section{Experimental}

\section{Materials}

Poly $(\gamma$-glutamic acid $)(\gamma$-PGA, MW $=700 \mathrm{kDa})$ was purchased from Shineking Biotechnology (Nanjing, China), imiquimod (IMQ) was obtained from meilunpharm (China), and ovalbumin (OVA, MW $=40 \mathrm{kDa}$ ) was purchased from Sigma. The reagents (1-dodecanol, DCM, DMSO, DMF, $\mathrm{SOCl}_{2}$, EtOH, $\mathrm{HCl}, \mathrm{Et}_{3} \mathrm{~N}, \mathrm{HOAc}$, and $\mathrm{Na}_{2} \mathrm{SO}_{4}$ ) were obtained from Aladdin, fluoresceinamine (5- $\left.\mathrm{NH}_{2}-\mathrm{FL}\right)$ was purchased from Asianchem (Nanjing, China). Doubly distilled water from a Millipore system (Milli-Q Academic A10) was used for all analysis and purification steps.

\section{Synthesis of $\gamma$-PGA-IMQ-LA-FL (FIP)}

Step-I. A $100 \mathrm{~mL}$ round-bottom flask was charged with poly $(\gamma$-glutamic acid) $(1.3 \mathrm{~g}, 10 \mathrm{mmol})$, dry DCM $(50 \mathrm{~mL})$ and a catalytic amount of DMF. The reaction mixture was cooled to $0{ }^{\circ} \mathrm{C}$ and stirred for 10 minutes. Then, $\operatorname{SOCl}_{2}(1.1 \mathrm{~mL}, 1.5$ equiv.) was added dropwise to the reaction mixture and stirred at room temperature for $12 \mathrm{~h}$. The resulting mixture was concentrated under reduced pressure to afford acid chloride, which was used directly without further purification for the next step.

Step-II. To a solution of IMQ ( $0.6 \mathrm{~g}, 0.25$ equiv.), fluoresceinamine ( $7 \mathrm{mg}, 0.002$ equiv.) and $\mathrm{Et}_{3} \mathrm{~N}$ (1.67 mL, 1.5 equiv.) in dry DMSO (20 mL), acid chloride ( $1.65 \mathrm{~g}, 1.0$ equiv.) was added dropwise at $0{ }^{\circ} \mathrm{C}$ and the reaction mixture was stirred at room temperature for $12 \mathrm{~h}$. Then 1-dodecanol (LA) ( $0.93 \mathrm{~g}, 0.5$ equiv) 
was added and kept for $12 \mathrm{~h}$. At last, water $(40 \mathrm{~mL})$ was added, the organic layer was separated and the aqueous layer was extracted with DCM $(4 \times 30 \mathrm{~mL})$. The combined organic layer was washed with water $(4 \times 30 \mathrm{~mL})$. After that, the organic layer was dried over $\mathrm{Na}_{2} \mathrm{SO}_{4}$ and concentrated under reduced pressure. The crude mass was purified by dialysis against a cellulose dialysis membrane $(\mathrm{MWCO}=3500 \mathrm{Da})$ against EtOH-water $(1: 1, \mathrm{v}-\mathrm{v})$ for 3 days to remove small molecular impurities, and then lyophilized as a pale yellow solid. Spectral data are in accordance with the reported data.

\section{Characterization of FIP}

The ${ }^{1} \mathrm{H}$ NMR spectra were recorded using a Bruker DMX 400 spectrometer with $\mathrm{D}_{2} \mathrm{O}$ or DMSO- $d_{6}$ solvent. UV-vis (UV-2700, SHIMADZU, Japan), fluorescence (F-4600, HITACHI, Japan) and FI-IR (ALPHA, Bruker, Germany) were used to analyzie IMQ and fluoresceinamine whether grafted to $\gamma$-PGA. The zeta potential values of FIP were analyzed using a Zetasizer-Nano (Malvern, UK).

\section{Animals and cells}

In vivo experiments were conducted using 6-8 week old female $\mathrm{BALB} / \mathrm{c}$ mice with weights of $18-20 \mathrm{~g}$ (purchased from the Comparative Medicine Center of Yangzhou University). Mice were housed in the laboratory animal facilities under specific pathogen-free conditions with a normal schedule and with free access to food and water. All experiments were strictly performed in accordance with the Institutional guidelines. RAW264.7 cells (ATCC) were cultured in high-glucose Dulbecco's modified Eagle's medium (DMEM, Transgen Biotech), supplemented with $10 \%$ fetal bovine serum (FBS, Gibco) and 1\% penicillin with $5 \%$ $\mathrm{CO}_{2}$ at $37{ }^{\circ} \mathrm{C}$.

\section{Safety study}

In vitro cytotoxicity of the FIP. In vitro cytotoxicity of the FIP was evaluated as described previously. ${ }^{35}$ RAW264.7 cells were first cultured in DMEM, then diluted to the final cell concentration of $2 \times 10^{6}$ cells per $\mathrm{mL}$, transferred to 96 -well plates at $100 \mu \mathrm{L}$ per well, and cultured at $37{ }^{\circ} \mathrm{C}$ for $24 \mathrm{~h}$. After discarding the supernatant in the wells, $100 \mu \mathrm{L}$ of FIP (diluted in DMEM culture at different concentrations) was added into the wells, followed by incubation at $37{ }^{\circ} \mathrm{C}$ for $24 \mathrm{~h}$. Then, $10 \mu \mathrm{L}$ of CCK-8 reagent (UE everbright) was added and incubated for $4 \mathrm{~h}$. The absorbance was read using a microplate reader (Epoch, BioTek, USA) at a wavelength of $450 \mathrm{~nm}$.

$$
\text { Cell viability }(\%)=\frac{A_{\text {sample }}}{A_{\text {control }}} \times 100 \%
$$

where $A_{\text {sample }}$ and $A_{\text {control }}$ are the absorbance values of the wells (FIP) and control wells (PBS), respectively. For each sample, the final absorbance was an average of those measured from six wells in parallel.

\section{Flow cytometry experiments}

To determine FIP uptake by the cells quantitatively, RAW264.7 cells were seeded in a 24 -well plate at a density of $2 \times 10^{5}$ cells per $\mathrm{mL}$ before the cells were pulsed with FIP at different concentrations. After $24 \mathrm{~h}$ of incubation, the macrophages were dissociated using PBS followed by centrifugation for $15 \mathrm{~min}$ at $1000 \mathrm{rpm}$ and resuspended with ice PBS for detection. Flow cytometry analysis was performed on a BD Accuri. Data were processed using the FlowJo software package.

\section{Confocal microscopy}

RAW264.7 cells were pulsed overnight at $37{ }^{\circ} \mathrm{C}$ with $40 \mu \mathrm{L}$ of a $1 \mathrm{mg} \mathrm{mL}{ }^{-1}$ FIP solution in PBS. Next, the culture medium was aspirated and washed with PBS three times. Thirdly, cells were fixed with 4\% paraformaldehyde (PFA) solution for $30 \mathrm{~min}$ followed by washing with PBS. Fourthly, the cells were stained with phalloidin (red, labeling cell membrane) in a dark environment at room temperature for $1 \mathrm{~h}$ and then washed with PBS three times. Finally, the DAPI (blue, labeling cell nucleus) dye was added using the same method for $15 \mathrm{~min}$, washed with PBS three times and fluorescently confocal imaged using an Ultra View VOX (PerkinElmer) microscope $(60 \times)$.

\section{Histological examination}

In vivo toxicity of FIP was evaluated by Histological examination in BALB/c mice. Briefly, the PBS-dissolved FIP $\left(500 \mu \mathrm{g} \mathrm{mL} \mathrm{m}^{-1}\right)$ was injected through hypodermic injection at a dose of $200 \mu \mathrm{L}$ per mouse $(n=3)$ and they were sacrificed 28 days later. The major organs (spleen, liver and kidneys) and the subcutaneous tissues at the injection sites were excised and fixed in 4\% PFA solution. Then, the tissues were embedded in paraffin, sectioned, and stained with hematoxylin and eosin. The histological sections were photographed under an Olympus microscope (Olympus IX71, Tokyo, Japan).

\section{Preparation of the related vaccines}

Protocol 1\#. Female BALB/c mice (18-20 g) were randomly divided into six groups with six mice in each group $(n=6)$. The simple mixture of fluidic OVA, FIP (OVA + FIP), $\gamma$-PGA $(\mathrm{OVA}+\gamma$-PGA) or IMQ (OVA + IMQ) in PBS solution was prepared as vaccine candidates, respectively. Mice were immunized via sub-cutaneous injections of each vaccine candidate, OVA, FIP or PBS, respectively (Table 1), and OVA-specific antibody levels in serum at 28 days post vaccination (dpv) were measured by ELISA.

Protocol 2\#. Different types of emulsion have been widely exploited in vaccines. Here, to further investigate whether FIP could have a synergistic effect with/in different types of emulsion, EAS (a patented oil-in-water emulsion in our laboratory), ISA201 (a commercial adjuvant for the preparation of wateroil-water emulsions) or mineral oil (a conventional adjuvant for

Table 1 Immunization groups of different compositions in the mice study

\begin{tabular}{llll}
\hline Group & Antigen & Dose/mouse & Form \\
\hline PBS & OVA $(100 \mu \mathrm{g})$ & $200 \mu \mathrm{L}$ & Water \\
OVA & - & $200 \mu \mathrm{L}$ & Water \\
FIP $\left(500 \mu \mathrm{g} \mathrm{mL}^{-1}\right)$ & OVA $(100 \mu \mathrm{g})$ & $200 \mu \mathrm{L}$ & Water \\
$\gamma$-PGA $\left(500 \mu \mathrm{g} \mathrm{mL}^{-1}\right)$ & OVA $(100 \mu \mathrm{g})$ & $200 \mu \mathrm{L}$ & Water \\
IMQ $\left(500 \mu \mathrm{g} \mathrm{mL}^{-1}\right)$ & OVA $(100 \mu \mathrm{g})$ & $200 \mu \mathrm{L}$ & Water \\
FIP $\left(500 \mu \mathrm{g} \mathrm{mL}^{-1}\right)$ & & Water
\end{tabular}


Table 2 Immunization groups of different formations in a mice study

the preparation of a water-in-oil emulsion) were chosen to prepare different vaccine formulations. The protocol has been described and published previously. ${ }^{36}$ Briefly, OVA or the mixture of OVA and FIP (OVA + FIP) (the concentration of OVA or FIP was the same as that in Protocol 1\#) was further mixed thoroughly with different adjuvant formulations (EAS, ISA201 or mineral oil) on a shaker. Consequently, we acquired the oil-in-water vaccine formulation (OVA-O/W) and (OVA + FIP-O/W), the water-oil-water vaccine formulation (OVA-W/O/W) and (OVA + FIP-W/O/W) and the water-in-oil vaccine formulation (OVA-W/O) and (OVA + FIP-W/O), respectively. Then female $\mathrm{BALB} / \mathrm{c}$ mice (18-20 g) were randomly divided into nine groups $(n=6)$ and immunized subcutaneously two times at two-week intervals with $200 \mu \mathrm{L}$ per dose. Mice immunized with the fluidic OVA alone, OVA + FIP or PBS, respectively, were used as controls (Table 2). Serum was collected at $14 \mathrm{dpv}$ and $28 \mathrm{dpv}$ for OVA-specific IgG measurement using ELISA.

\section{Protocol 3\#}

To reduce the cost of the vaccine, we investigated the dosage effect of FIP on OVA-specific IgG. Three groups of OVA + FIP-O/W vaccines with different concentrations $\left(5,100\right.$ or $\left.500 \mu \mathrm{g} \mathrm{mL}{ }^{-1}\right)$ OVA-O/W, the fluidic OVA and PBS as the control groups were carried out to assay OVA-specific IgG. Female BALB/c mice $(n=6)$ were immunized twice in the six groups as shown in Table 3.

\section{OVA-specific IgG antibody analysis}

Serum was collected 14 days and 28 days after the first immunization. OVA-specific IgG antibody levels were determined using an enzyme-linked immunosorbent assay (ELISA). Briefly, a 96-well ELISA plate was coated with $100 \mu \mathrm{L}$ of $10 \mu \mathrm{g} \mathrm{mL}^{-1}$ OVA antigen in carbonate buffer $(\mathrm{pH} 9.6)$ at $4{ }^{\circ} \mathrm{C}$ overnight and then blocked with blocking buffer (PBS containing 5\% BSA, pH 7.4) at $37{ }^{\circ} \mathrm{C}$ for $2 \mathrm{~h}$. After washing with PBST (PBS containing $0.1 \%$ tween-20, pH 7.4), serially diluted serum was added into each well

Table 3 Immunization groups of different concentrations of OVA + FIP$\mathrm{O} / \mathrm{W}$ in the mice study

\begin{tabular}{|c|c|c|c|c|}
\hline Group & & Antigen & Dose/mouse & Form \\
\hline PBS & & - & $200 \mu \mathrm{L}$ & Water \\
\hline OVA & & OVA $(100 \mu \mathrm{g})$ & $200 \mu \mathrm{L}$ & Water \\
\hline \multirow[t]{3}{*}{ FIP } & $5 \mu \mathrm{g} \mathrm{mL}^{-1}$ & OVA $(100 \mu \mathrm{g})$ & & $\mathrm{O} / \mathrm{W}$ \\
\hline & $100 \mu \mathrm{g} \mathrm{mL}^{-1}$ & OVA $(100 \mu \mathrm{g})$ & $200 \mu \mathrm{L}$ & $\mathrm{O} / \mathrm{W}$ \\
\hline & $500 \mu \mathrm{g} \mathrm{mL}^{-1}$ & 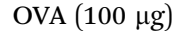 & & $\mathrm{O} / \mathrm{W}$ \\
\hline
\end{tabular}

and then incubated for $1.5 \mathrm{~h}$ at $37^{\circ} \mathrm{C}$. After washing with PBST five times, the plates were incubated with anti-mouse IgG-HRP antibody for another hour at $37{ }^{\circ} \mathrm{C}$ and then developed with TMB substrate for $30 \mathrm{~min}$. The reaction was terminated by adding $2 \mathrm{M}$ $\mathrm{H}_{2} \mathrm{SO}_{4}$, and then the absorbance at $450 \mathrm{~nm}$ was recorded using a microplate reader.

\section{Statistical analysis}

Data for cell viability, antibody and cytokine secretion assay were presented as mean \pm SD and were statistically analyzed using the two-tailed Student's t test. ${ }^{*} p<0.05,{ }^{* *} p<0.01$ was considered to be significant.

\section{Results and discussion}

\section{Polyglutamate conjugation of a long carbon-chain and IMQ (TLR7 agonist)}

$\gamma$-PGA is a kind of environmentally-friendly macromolecular substance with hydrophilicity, easy biodegradation and strong absorbability. Furthermore, the hydrophilic bioinspired synthetic polymer with an abundancy of carboxylic acid groups makes it is well suited for the design of water-soluble sustained release agents and drug carriers. ${ }^{19,20} \gamma$-PGA has already been employed in a phase III trial polymer-anticancer drug conjugate, Opaxio, consisting of paclitaxel conjugated to glutamic acid residues applied in ovarian or fallopian tube cancer treatment. ${ }^{37}$ An ideal adjuvant needs to dissolve in water and/or oil. To circumvent these issues De Geest's group designed lipid-polymer amphiphiles consisting of a lipid motif as the hydrophobic part and a hydrophilic polymer connected to the lipid moiety at one of its chain ends. ${ }^{15}$ With these in our minds, we conjugated IMQ, a small-molecule TLR7 agonist, and an LA motif as the hydrophobic part onto the $\gamma$-PGA polymer backbone to gain an amphiphilic polymer. This concept is illustrated in Scheme 2.

Amphiphilic FIP was synthesized by the reaction of $\gamma$-PGA with $\mathrm{SOCl}_{2}$ to generate a reactive intermediate of acyl chloride polymer backbone that can later on be used for conjugation of IMQ, LA, as well as for fluorescent labelling. The purified FIP was characterized in DMDO- $d_{6}$ using ${ }^{1} \mathrm{H}$ NMR spectroscopy. From Fig. 1, we can see that the IMQ (in $\mathrm{D}_{2} \mathrm{O}+\mathrm{CF}_{3} \mathrm{CO}_{2} \mathrm{D}$, blue box) and LA (in $\mathrm{D}_{2} \mathrm{O}$, red box) were attached to $\gamma$-PGA units via
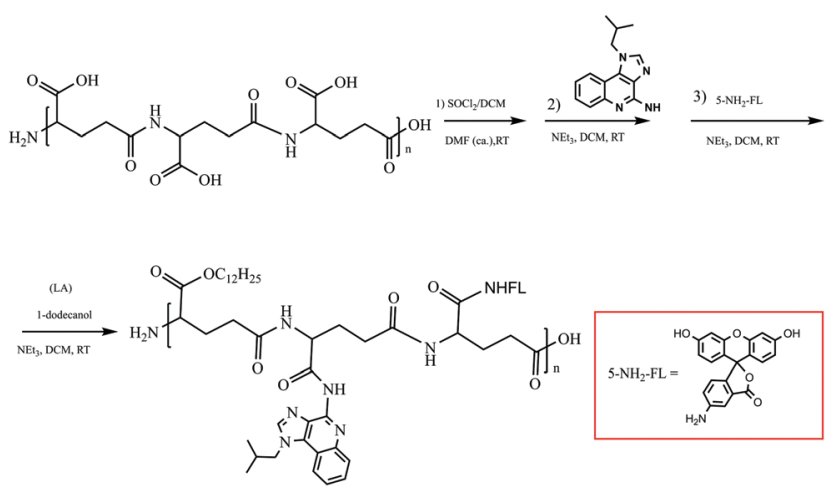

Scheme 2 The synthesis route to FIP. 


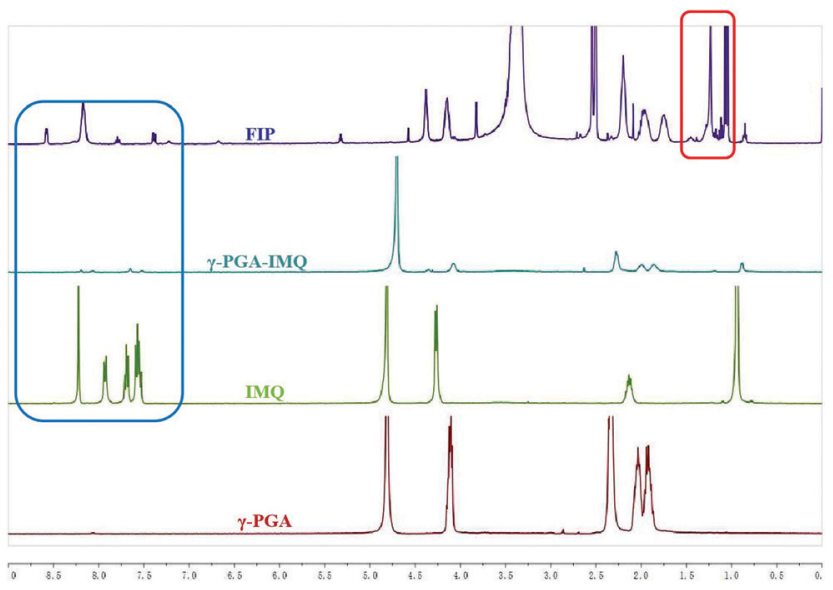

Fig. $1{ }^{1} \mathrm{H}$ NMR image of FIP, $\gamma$-PGA-IMQ, IMQ and $\gamma$-PGA.

covalent bonds. The degree of substitution of the IMQ was determined from the ratio between the integrated area resonances of the hydrogen at the $\mathrm{C}-2$ position (Scheme 1) in the $\gamma$-PGA (in $\mathrm{D}_{2} \mathrm{O}$ ) groups $(\delta 4.15 \mathrm{ppm}$ ) and the aromatic ring hydrogen in IMQ units ( $\delta 6.68 \mathrm{ppm})$. Likewise, the degree of esterification with LA was calculated using the ratio between the integrated area resonances of the hydrogen at the C-12 in the LA methyl group $(\delta 0.85 \mathrm{ppm})$ and the hydrogen at $\mathrm{C}-2$ $(\delta 4.15 \mathrm{ppm})$ in the $\gamma$-PGA units. The results illuminated that the grafting degrees of IMQ and LA were about $8 \%$ and $5.6 \%$ in mole $\%(12 \%$ and $6 \%$ in wt $\%)$, respectively. Due to the content of fluorescein being very low, its peaks barely show in ${ }^{1} \mathrm{H}$ NMR (Single ${ }^{1} \mathrm{H}$ NMR in Fig. S1, ESI $\dagger$ ).

To investigate whether IMQ and fluorescein conjugated to the $\gamma$-PGA framework, we carried out spectroscopic studies to examine the effectiveness of FIP. The UV-vis spectrum of FIP showed distinct IMQ peaks at about 306 and $318 \mathrm{~nm}$ (Fig. 2a). The absorbance from 400 to $530 \mathrm{~nm}$ was ascribed to fluorescein absorbance. To further verify the grafting of IMQ and fluorescein to the $\gamma$-PGA framework, a fluorescence assay was (a)

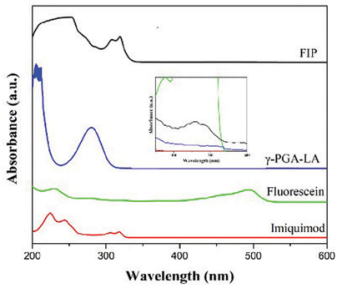

(c)

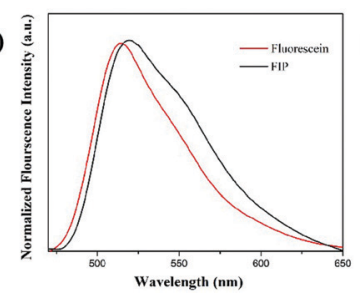

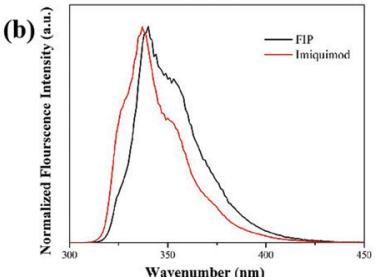

(d)

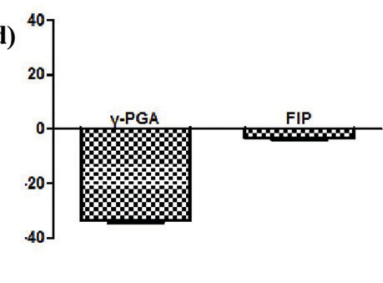

Fig. 2 (a) UV-Vis absorbance spectra. The fluorescence spectra of FIP with Ex $280 \mathrm{~nm}$ (b) and Ex $455 \mathrm{~nm}$ (c) for IMQ, fluorescein and FIP, and (d) the zeta potential value.

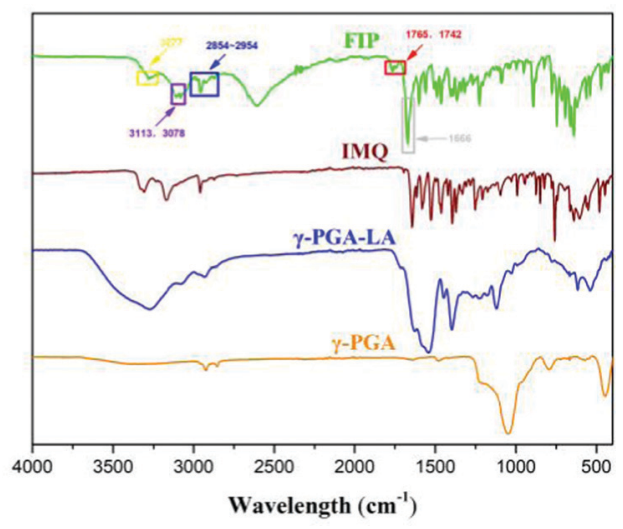

Fig. 3 The FI-IR images of FIP and related substrates.

conducted with Ex 280 (Fig. 2b) and $455 \mathrm{~nm}$ (Fig. 2c) for IMQ and fluorescein, respectively. The results suggested that IMQ and fluorescein were successfully graft to the $\gamma$-PGA framework.

As shown in Fig. 2d, the hydrodynamic diameter of FIP was about $284.7 \mathrm{~nm}$ with a polydispersity index (PDI) of 0.265 (Fig. S2, ESI $\dagger$ ). The low PDI indicates excellent and uniform dispersion of FIP. The zeta potential value of FIP was $-4.00 \pm$ $0.91 \mathrm{mV}$, while the value for $\gamma$-PGA was $-35.10 \pm 1.49 \mathrm{mV}$, which suggests that $\gamma$-PGA was successfully conjugated with IMQ and fluorescein.

Fourier-transform infrared (FT-IR) spectroscopy was used to confirm the IMQ and LA grafting to the $\gamma$-PGA skeleton (Fig. 3). Compared with $\gamma$-PGA, the absorption peaks at 3078 and $3113 \mathrm{~cm}^{-1}$ (purple box) were assigned as $\mathrm{sp}^{2}$ hybridization $\mathrm{C}-\mathrm{H}$ stretching from the IMQ aromatic ring. The peak at $3277 \mathrm{~cm}^{-1}$ was ascribed to the $\mathrm{N}-\mathrm{H}$ vibration of IMQ. The peaks at 1742 and $1765 \mathrm{~cm}^{-1}$ (red box) were ascribed to the $\mathrm{C}=\mathrm{O}$ stretching of an ester. The peaks between $2854-2954 \mathrm{~cm}^{-1}$ (blue box) were enhanced due to $\mathrm{sp}^{3}$ hybridization $\mathrm{C}-\mathrm{H}$ stretching of the LA chain. The new peak at $1666 \mathrm{~cm}^{-1}$ (gray box) compared with that of crude $\gamma$-PGA was due to amide carbonyl stretching vibration, in which IMQ reacted with $\gamma$-PGA. These results demonstrate that IMQ and LA were successfully modified onto $\gamma$-PGA (single FI-IR of FIP and $\gamma$-PGA-LA is shown in Fig. S3, ESI $\dagger$ ).

\section{Cellular cytotoxicity}

An ideal vaccine adjuvant should have excellent biocompatibility and safety. The good cell viability of FIP was evidenced by the CCK-8 assay. RAW264.7 cells were treated with different concentrations $\left(5,100\right.$ or $\left.1000 \mu \mathrm{g} \mathrm{mL}{ }^{-1}\right)$ of FIP (containing about $12 \%$ IMQ, w/w) or IMQ (100 or $1000 \mu \mathrm{g} \mathrm{mL} \mathrm{m}^{-1}$ ), acetic acid (HOAc, pH 6), and PBS for 24 h. As shown in Fig. 4, the FIP hardly caused a loss in the proliferation ability of the cells and the survival rate of the cells treated with FIP ( 5 or $\left.100 \mu \mathrm{g} \mathrm{mL} \mathrm{m}^{-1}\right)$ had no significant difference to that of the PBS-treated cells $(p>0.05)$. However, the cell viabilities showed a significant decline when treated with plain IMQ acid solution $(\mathrm{pH} 6)$ at the concentration of $100 \mu \mathrm{g} \mathrm{mL}{ }^{-1}$, or HOAc solution $(p<0.05)$. IMQ demonstrated high toxicity to RAW264.7 cells at $100 \mu \mathrm{g} \mathrm{mL} \mathrm{mL}^{-1}$ (cell viability $<17 \%$ ). 


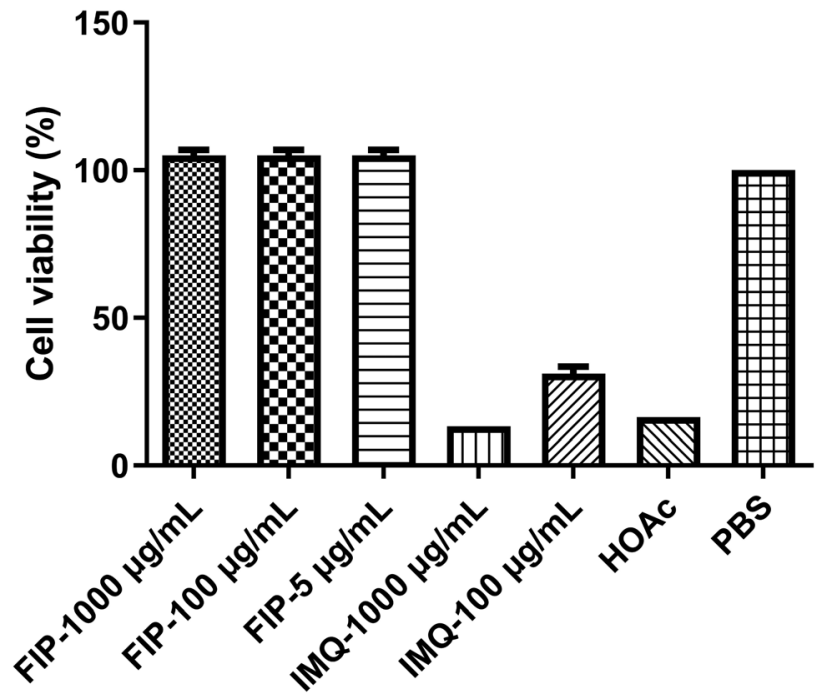

Fig. 4 The cell viability of each sample with different concentrations.

Therefore, the FIP could be considered as a nontoxic, safetyacceptable and biocompatible adjuvant.

Pure IMQ has been reported to cause many adverse reactions, such as local skin inflammation, erosion and ulcers when injected into skin. ${ }^{38,39}$ Also, IMQ can dissolve in acid solution, then further stimulate the body and pose some safety risks. An effective solution to the problem is covalent ligation of IMQ to a larger carrier, which prevents uncontrolled systemic distribution. ${ }^{40}$ As shown in Fig. 5, after subcutaneous injection of mice with FIP $\left(1000 \mu \mathrm{g} \mathrm{mL}^{-1}\right)$ or IMQ $\left(100 \mu \mathrm{g} \mathrm{mL} \mathrm{L}^{-1}\right)$, the IMQ group showed significant skin reactions after 2 days, however, no adverse skin reaction was observed in the FIP group up to 14 days after immunization. The FIP did not trigger any skin redness, swelling or ulceration. The mice in the FIP group were fine with a normal diet and daily routine.

\section{Histological examination}

To further evaluate the toxicity of FIP in vivo, histological examination was performed using the standard hematoxylin

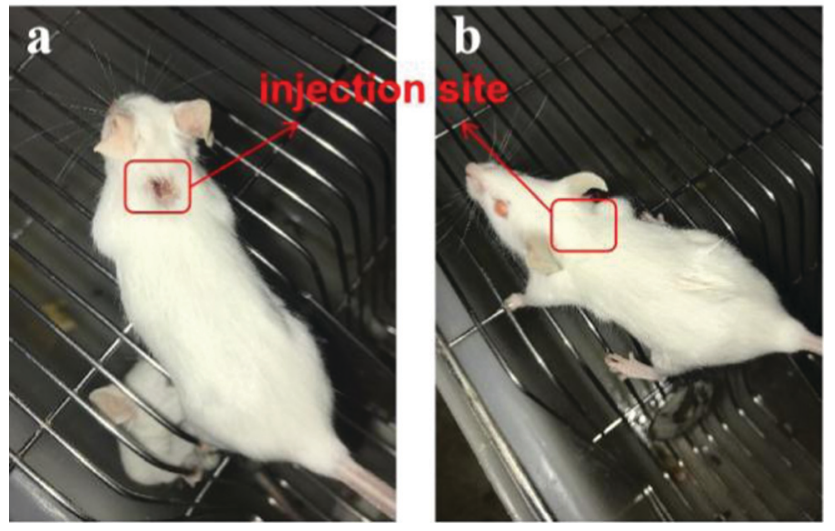

Fig. 5 The skin reaction pf mice subjected to subcutaneous injection with (a) IMQ and (b) FIP in PBS solution after 2 days.

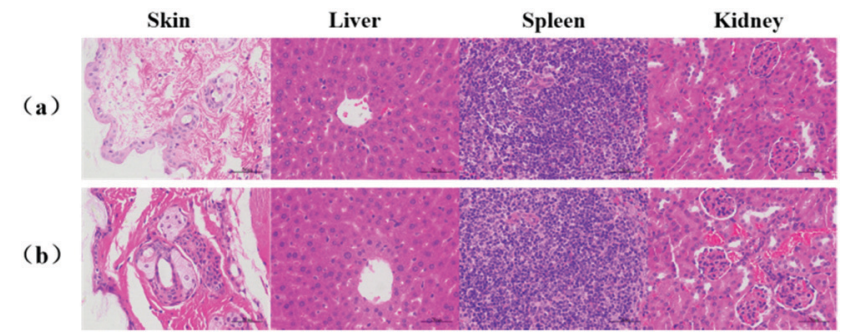

Fig. 6 HEE staining images from the major organs and injection sites of BALB/C mice after 28 days of immunization. Data are representative of at least 3 mice. The scale bar is $50 \mu \mathrm{m}$.

and eosin (H\&E) staining method. The FIP were injected through hypodermic injection at a concentration of $500 \mu \mathrm{g} \mathrm{mL}$ $(200 \mu \mathrm{L})$ per mouse $(n=3)$ for $28 \mathrm{~d}$, and then the mice were sacrificed and the major organs (spleen, liver and kidneys) and the subcutaneous tissues at the injection sites were removed and taken out for pathological analysis. The histopathological alterations were shown in Fig. 6. For the FIP group mice, the spleen, liver, kidneys and the subcutaneous tissues at the injection sites showed no marked pathological abnormalities and adverse effects as compared to the control group (Fig. 6b). The results suggest that the FIP adjuvant-based vaccine has good safety.

\section{Cellular uptake measurement by flow cytometry}

The activation or polarization of macrophages is an integral part in innate and adaptive immune responses. ${ }^{41,42}$ Macrophages can be polarized into M1 (classically activated) and M2 (alternatively activated) phenotypes. Activated macrophages may be induced by various cytokines. ${ }^{43}$ To further qualitatively evaluate the effect of FIP on RAW 264.7 activation, the concentration of cytokines in the supernatants of RAW264.7 cells stimulated with OVA or OVA + FIP was measured by ELISA kits. The results showed that FIP significantly promoted the secretion of inflammatory cytokines IL-2, 4, 6 and IFN- $\gamma$, which may be an important indicator for M1 macrophage activation (Fig. S4, ESI $\dagger$ ).

To investigate the cellular uptake of FIP by RAW264.7 cells, a flow cytometer assay was conducted. The median fluorescence intensity (MFI) of cells treated with different concentrations of FIP was determined. As shown in Fig. 7, RAW 264.7 cells incubated with FIP showed a high fluorescence intensity, which indicated that FIP can be engulfed effectively by RAW

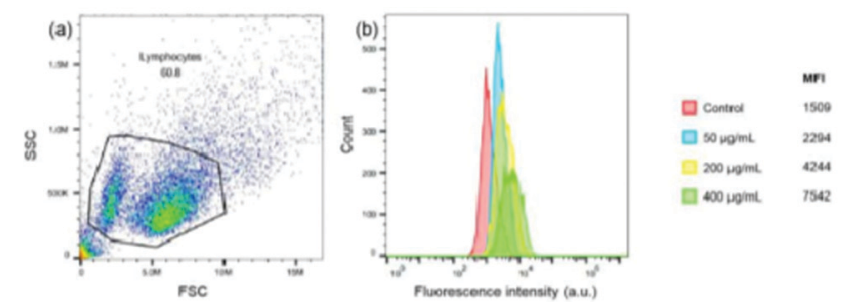

Fig. 7 Flow cytometry analysis of RAW264.7 cell uptake of FIP. (a) Aspect ratio; (b) MFI image of RAW264.7 cells incubated with different FIP concentrations. 
(a)
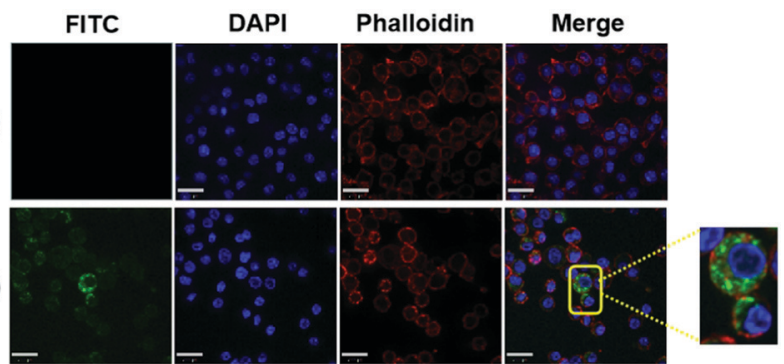

Fig. 8 Confocal microscopy images of RAW264.7 cells incubated with (a) DMEM and (b) FIP (40 $\left.\mu \mathrm{L}, 1 \mathrm{mg} \mathrm{mL}^{-1}\right)$.

264.7 cells. The data in Fig. 7b also demonstrate that the cellular uptake of FIP by RAW 264.7 cells (the percentages of fluorescent cells) increased in a dose dependent manner.

To corroborate the uptake of FIP, the cellular localization of FIP in RAW264.7 cells was also observed by confocal microscopy. Cells were stained with DAPI (for cell nucleus) and Phalloidin (for membrane), respectively. Images of the RAW264.7 cells without (Fig. 8a) and with FIP (Fig. 8b) show that FIP did not cause significant cytotoxicity and did not cause cells apoptosis in RAW264.7 cells. Meanwhile, the green fluorescein signals could only be obtained in RAW264.7 cells incubated with FIP. This indicated that FIP could be engulfed by RAW264.7 cells and packaged in the cell membrane, which would be beneficial for antigen delivery.

\section{In vivo effect of FIP on the immune system}

After verifying the improved safety profile as well as the efficiency of cell uptake with FIP in vitro and in vivo, we then evaluated the adaptive immune responses in mice immunized with different components, formulations and concentrations.

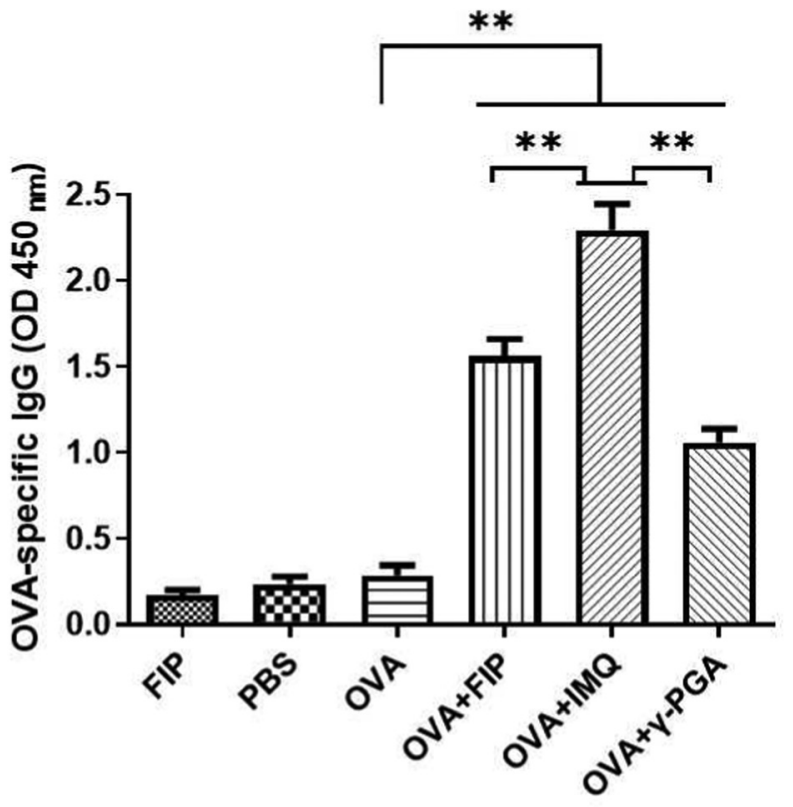

Fig. 9 The OVA special-lgG of mice immunized different components of FIP in PBS solution.
IMQ has been used as a small molecule immunomodulator in anti-virus and anti-tumor treatments. To elucidate the possible adjuvant properties of FIP, we evaluated the immunostimulating efficacy of FIP, IMQ or $\gamma$-PGA with OVA as a model antigen in PBS solution.

With respect to the humoral responses, IMQ, which was the main framework for immune stimulation, induced the strongest serum OVA-specific IgG among those groups (Fig. 9). The dose of vaccine needs to be determined in the practical use process. Therefore, we chose the protocol of keeping the fixed adjuvant dosage (Table 1). Mice immunized with OVA + IMQ appeared to have obvious redness, swelling, festering at the injection sites and other adverse reactions (Fig. 5a). However, there were no side-effects when using OVA + IMQ (Fig. 5b). This phenomenon may be attributed to the fact that IMQ in an unformulated state rapidly distributes throughout the whole body and causes systemic inflammation. Covalent conjugation of IMQ to macromolecular $\gamma$-PGA carriers strongly abrogates systemic dissemination due to controlled release of IMQ. ${ }^{44}$ Interestingly, as shown in Fig. 9, OVA + FIP demonstrated a significantly stronger immune response than its backbone OVA $+\gamma$-PGA but less than OVA + IMQ $(p<0.05)$. The results indicated that IMQ can effectively enhance the immune response. According to ${ }^{1} \mathrm{H}$ NMR (Fig. S1a, ESI $\dagger$ ) the content (mass ratio) of IMQ in FIP was about 12\%. $500 \mu \mathrm{g}$ of FIP (containing approximately $60 \mu \mathrm{g}$ of IMQ) had a slightly lower immune-stimulating potentiality than that of $500 \mu \mathrm{g}$ pristine IMQ. As an amphiphilic polymer, FIP can self-assemble into nanoparticles (ANPs). Particles with a size of 20-200 nm undergo endocytosis and are preferentially ingested by dendritic cells (DCs). ${ }^{45,46}$ In this study, we obtained an average Z-average diameter of about $284.7 \mathrm{~nm}$ (Fig. S2, ESI $\dagger$ ). Owing to an increase in the surface hydrophobicity of ANPs, an enhanced adhesion force between ANPs and cell membranes facilitated internalization of FIP and antigens into cells. Furthermore, ANPs were drained into lymphoid tissue, ${ }^{47}$ degraded to release IMQ slowly, and enhanced immune reactions. Emulsions are liquid dispersions of two immiscible phases and have a long history of use as adjuvants in both human and veterinary vaccines. ${ }^{48}$ There are mainly three types of emulsion vaccines according to the inner water phase or outer water phase: water-in-oil (W/O), oil-inwater $(\mathrm{O} / \mathrm{W})$ and water-oil-water $(\mathrm{W} / \mathrm{O} / \mathrm{W})$. Due to the reactogenicity of $\mathrm{W} / \mathrm{O}$ emulsions (traditional vaccines), $\mathrm{O} / \mathrm{W}$ or $\mathrm{W} / \mathrm{O} / \mathrm{W}$ emulsions are considered as better alternatives. However, the latter two types of emulsions are evaluated as less effective in affording high antibody levels and long antibody duration. Incorporating molecular immunopotentiators within emulsions could overcome the drawbacks of molecular adjuvants (such as biodistribution and pharmacokinetics) and thus improve the adjuvanticity of emulsions.

We next determined whether FIP would exert any adaptive immune enhancing effects when incorporated into different emulsions. Mice in each group were immunized with OVA + FIP-O/W, OVA + FIP-W/O/W, OVA + FIP-W/O, OVA-O/W, OVA-W/ $\mathrm{O} / \mathrm{W}$, OVA-W/O, OVA alone, OVA + FIP or PBS, respectively. Sera samples were collected at 14 and $28 \mathrm{dpv}$ after the first immunization and OVA-specific IgG was detected by ELISA 


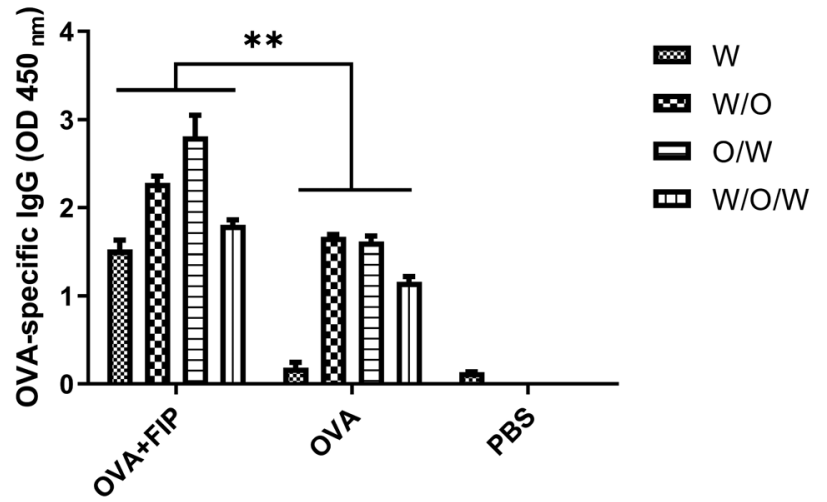

Fig. 10 The OVA specific-lgG of mice immunized with different formulations at $28 \mathrm{dpv}$.

(Fig. S5, ESI $\dagger$ and Fig. 10). As shown in Fig. 10, all emulsion formulations elicited significantly higher humoral immune responses than that of OVA alone at $28 \mathrm{dpv}(p<0.05)$. The OVA + FIP-O/W and OVA + FIP-W/O vaccinated groups had significantly higher OVA-specific antibodies than those of the different formations of OVA + FIP vaccinated groups at 2 weeks after the first and second immunizations $(p<0.05)$. Furthermore, various OVA + FIP emulsion formulations induced significantly enhanced OVA-specific antibodies compared to OVA-W/O/W, OVA-O/W or OVA-W/O. The results suggested that incorporation of FIP into $\mathrm{O} / \mathrm{W}$ or $\mathrm{W} / \mathrm{O}$ emulsion formulations could significantly improve the adjuvanticity of these emulsions.

Interestingly, compared with the different formulations of pure OVA, our material has a stronger immune stimulating potentiality. After 28 days of immunization to determine the $\mathrm{OD}_{450} 0_{\mathrm{nm}}$ of OVA + FIP comparison by ELSIA it can be found that $\mathrm{O} / \mathrm{W}>\mathrm{W} / \mathrm{O}>\mathrm{W} / \mathrm{O} / \mathrm{W} \approx \mathrm{W}$. The data

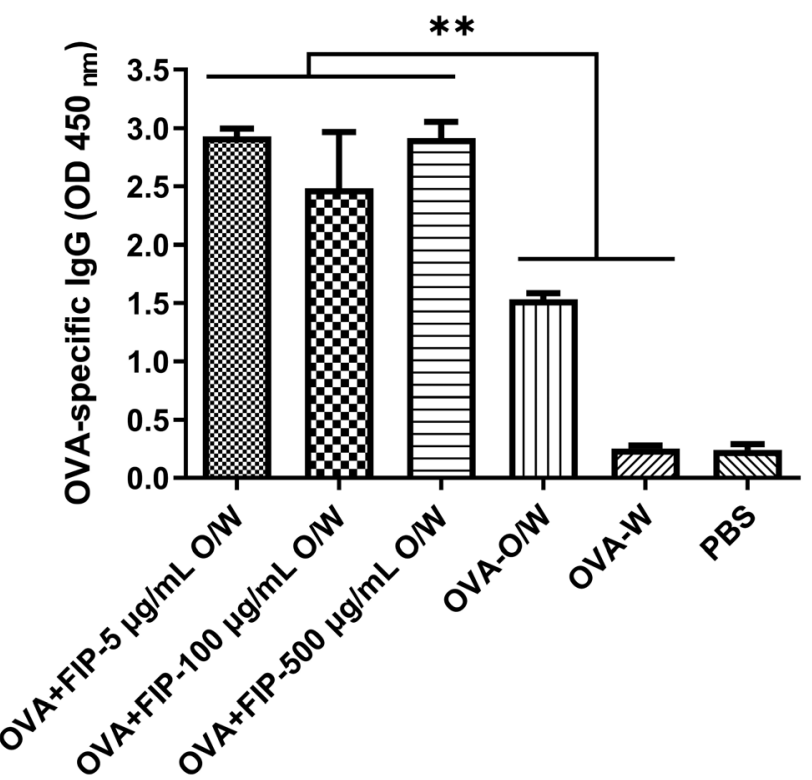

Fig. 11 The OVA specific-IgG of mice immunized with OVA + FIP-O/W at different concentrations in $28 \mathrm{dpv}$. indicate that the FIP material could be applied to multiformulations as an ideal adjuvant, especially as a water formulation adjuvant.

Considering the practical application of FIP adjuvant, we then tested the effect of adjuvant dose dependence on immune potentiality. Fig. 11 shows the immune responses induced by different adjuvant dosages for OVA, and the results demonstrate that the OVA antibody titer was the highest when the concentration of FIP was $5 \mu \mathrm{g} \mathrm{mL}^{-1}$ compared with other higher concentrations of FIP. The experiments indicate that low doses of FIP were adequate for eliciting effective immune responses. Future comprehensive work involving the incorporation of FIP into other antigens or formulations for different animals are needed to obtain a synergetic effect.

\section{Conclusions}

In summary, we report a simple yet efficient amphiphilic anionic FIP as a vaccine adjuvant for potent immunostimulatory effects after subcutaneous administration. Flow cytometry analysis of RAW264.7 cells demonstrated that FIP can be effectively taken up by the cells. Confocal fluorescent images further confirmed FIP distributed into the cell membrane. The CCK-8 assay and H\&E staining revealed that FIP had low toxicity and good biocompatibility. These results indicate that the FIP adjuvant may have good safety in vivo. Low dose $\left(5 \mu \mathrm{g} \mathrm{mL}{ }^{-1}\right)$ in oil-in-water $(\mathrm{O} / \mathrm{W})$ formulation can induce high immune responses and enhanced higher OVA-specific antibodies. Furthermore, the FIP maintained long-time immune memory and lasting immunity effectivity. The FIP provided a novel protein-based vaccine delivery platform, which was capable of inducing strong humoral and cellular immunity for effective vaccine prevention. Controlling the pharmacokinetics of IMQ through conjugation to the $\gamma$-PGA skeleton, therefore, enables the development of potent adjuvants in vaccines to drive durable, high-quality immunity responses and long immune memory with strongly reducing systemic toxicity of IMQ. We believe that the strategy explored here will be facile and effective toward generating subunit vaccines for improving the immune stimulating effect and will have immense potentiality in the development of immunotherapy for various diseases.

\section{Author contributions}

Synthesis (W. Z. Yin). Characterization (W. Z. Yin and D. C. Xuan). Animals and cells (W. Z. Yin, D. C. Xuan, F. Ma, B. H. Deng and M. X. Zhou). Supervision (Y. Lu and J. Q. Zhang). Writing - original draft (W. Z. Yin and J. Q. Zhang). Writing review \& editing (W. Z. Yin, J. Q. Zhang and Y. Lu).

\section{Conflicts of interest}

The authors declare no competing financial interest. 


\section{Acknowledgements}

This work was supported by the National Natural Science Foundation of China (No. 32172895, No. 31772701, No. 31902243) and the Special Fund for Independent Innovation of Agricultural Science and Technology in Jiangsu Province of China (No. CX (17) 3027).

\section{Notes and references}

1 M. C. Hanson, M. P. Crespo, W. Abraham, K. D. Moynihan, G. L. Szeto, S. H. Chen, M. B. Melo, S. Muerller and D. J. Irvine, J. Clin. Invest., 2015, 125(6), 2532-2546.

2 S. Plotkin, Proc. Natl. Acad. Sci. U. S. A., 2014, 111(34), 12283-12287.

3 H. J. Larson, C. Jarrett, E. Eckersberger, D. M. Smith and P. Paterson, Vaccine, 2014, 32(19), 2150-2159.

4 K. Hoelzer, L. Bielke, D. P. Blake, E. Cox, S. M. Cutting, B. Devriendt, E. Erlacher-Vindel, E. Goossens, K. Karaca, S. Lemiere, M. Metzner, M. Raicek, M. C. Suriñach, N. M. Wong, C. Gay and F. V. Immerseel, Vet. Res., 2018, 49, 70 .

5 S. Sevimli, F. C. Knight, P. Gilchuk, S. Joyce and J. T. Wilson, ACS Biomater. Sci. Eng., 2017, 3(2), 179-194.

6 L. Yang, W. Li, M. Kirberger, W. Z. Liao and J. Y. Ren, Biomater. Sci., 2016, 4, 785-802.

7 S. G. Reed, M. T. Orr and C. B. Fox, Nat. Med., 2013, 19, 1597-1608.

8 M. L. Mbow, E. D. Gregorio, N. M. Valiante1 and R. Rappuoli, Curr. Opin. Immunol., 2010, 22(3), 411-416.

9 A. Comberlato, K. Paloja and M. M. C. Bastings, J. Mater. Chem. B, 2019, 7, 6321-6346.

10 B. J. Ignacio, T. J. Albin, A. P. Esser-Kahn and M. Verdoes, Bioconjugate Chem., 2018, 29(3), 587-603.

11 A. Iwasaki and R. Medzhitov, Science, 2010, 327(5963), 291-295.

12 J. Kim, T. S. Griffith and J. Panyam, J. Pharmacol. Exp. Ther., 2019, 370(3), 715-724.

13 P. Redondo, J. del Olmo, A. Lopez-Diaz de Cerio, S. Inoges, M. Marquina, I. Melero and M. Bendandi, J. Invest. Dermatol., 2007, 127(7), 1673-1680.

14 J. P. Vasilakos and M. A. Tomai, Expert Rev. Vaccines, 2013, 12, 809-819.

15 J. D. Vrieze, B. Louage, K. Deswarte, Z. F. Zhong, R. D. Coen, S. V. Herck, L. Nuhn, C. K. Frich, A. N. Zelikin, S. Lienenklaus, N. N. Sanders, B. N. Lambrecht, S. A. David and B. G. D. Geest, Angew. Chem., Int. Ed., 2019, 58(43), 15390-15395.

16 L. Nuhn, N. Vanparijs, A. De Beuckelaer, L. Lybaert, G. Verstraete, K. Deswarte, S. Lienenklaus, N. M. Shukla, A. C. D. Salyer, B. N. Lambrecht, J. Grooten, S. A. David, S. D. Koker and B. G. D. Geest, Proc. Natl. Acad. Sci. U. S. A., 2016, 113(29), 8098-8103.

17 T. Y.-H. Wu, M. Singh, A. T. Miller, E. De Gregorio, F. Doro, U. D’Oro, D. A. G. Skibinski, M. L. Mbow, S. Bufali, A. E. Herman, A. Cortez, Y. K. Li, B. P. Nayak, E. Tritto,
C. M. Filippi, G. R. Otten, L. A. Brito, E. Monaci, C. Li, S. Aprea, S. Valentini, S. Calabró, D. Laera, B. Brunelli, E. Caproni, P. Malyala, R. G. Panchal, T. K. Warren, S. Bavari, D. T. O'Hagan, M. P. Cooke and N. M. Valiante, Sci. Transl. Med., 2014, 6(263), 263 ra160.

18 N. Pippa, M. Gazouli and S. Pispas, Vaccines, 2021, 9(6), 558. 19 S. H. Kelly, L. S. Shores, N. L. Votaw and J. H. Collier, Adv. Drug Delivery Rev., 2017, 114, 3-18.

20 W. G. Kim, B. Choi, H.-J. Yang, J.-A. Han, H. Jung, H. Cho, S. Kang and S. Y. Hong, Bioconjugate Chem., 2016, 27, 2007-2013.

21 A. Seth, M. B. Heo and Y. T. Lim, Biomaterials, 2014, 35, 7992-8001.

22 Y. H. Lin, C. K. Chung, C. T. Chen, H. F. Liang, S. C. Chen and H. W. Sung, Biomacromolecules, 2005, 6(2), 1104-1112.

23 T. Uto, M. Toyama, Y. Nishi, T. Akagi, F. Shima, M. Akashi and M. Baba, Results Immunol., 2013, 3, 1-9.

24 F. Shima, T. Uto, T. Akagi, M. Baba and M. Akashi, Acta Biomater., 2013, 9, 8894-8901.

25 M. Henriksen-lacey, K. S. Korsholm, P. Andersen, Y. Perrie and D. Christensen, Expert Opin. Drug Delivery, 2011, 8(4), 505-519.

26 M. Mockey, E. Bourseau, V. Chandrashekhar, A. Chaudhuri, S. Lafosse, E. Le Cam, V. F. J. Quesniaux, B. Ryffel, C. Pichon and P. Midoux, Cancer Gene Ther., 2007, 14(9), 802-814.

27 J. Wang, H. Zope, M. A. Islam, J. Rice, S. Dodman, K. Lipert, Y. H. Chen, B. R. Zetter and J. J. Shi, Front Bioeng. Biotech., 2020, 8, 787.

28 B. Wang, S. V. Herck, Y. Chen, X. Y. Bai, Z. F. Zhong, K. Deswarte, B. N. Lambrecht, N. N. Sanders, S. Lienenklaus, H. W. Scheeren, S. A. David, F. Kiessling, T. Lammers, B. G. De Geest and Y. Shi, J. Am. Chem. Soc., 2020, 142, 12133-12139.

29 C. S. Liong, A. A. A. Smith, J. L. Mann, G. A. Roth, E. C. Gale, C. L. Maikawa, B. S. Ou and E. A. Appel, Adv. Ther., 2021, 4(4), 2000081.

30 J. S. Wu, J. X. Li, N. Shu, Q. J. Duan, Q. S. Tong, J. Y. Zhang, Y. C. Huang, S. Y. Yang, Z. B. Zhao and J. Z. Du, Nano Res., 2022, 15, 510-518.

31 S. M. Jin, S. N. Lee, J. E. Kim, Y. J. Yoo, C. Song, H. S. Shin, H. Phuengkham, C. H. Lee, S. H. Um and Y. T. Lim, Adv. Sci., 2021, 8, 2102043.

32 S. B. Park, M. H. Sung, H. Uyama and D. K. Han, Prog. Polym. Sci., 2021, 113, 101341.

33 D. V. Lysebetten, A. Malfanti, K. Deswarte, K. Koynov, B. Golba, T. T. Ye, Z. F. Zhong, S. Kasmi, A. Lamoot and Y. Chen, ACS Appl. Mater. Interfaces, 2021, 13, 6011-6022.

34 I. W. Hamley, Bioconjugate Chem., 2021, 32, 1472-1490.

35 C. C. Meng, X. Zhi, C. Li, C. F. Li, Z. Y. Chen, X. S. Qiu, C. Ding, L. J. Ma, H. M. Lu, D. Chen, G. Q. Liu and D. X. Cui, ACS Nano, 2016, 10(2), 2203-2213.

36 J. Q. Zhang, J. F. Miao, X. G. Han, Y. Lu, F. Lv, B. H. Deng, Y. H. Zhao, C. Ding and J. B. Hou, BMC Vet. Res., 2018, 14, 415.

37 L. Paz-Ares, H. Ross, M. O’Brien, A. Riviere, U. Gatzemeier, J. Von Pawel, E. Kaukel, L. Freitag, W. Digel, H. Bischoff, 
R. García-Campelo, N. Iannotti, P. Reiterer, I. Bover, J. Prendiville, A. J. Eisenfeld, F. B. Oldham, B. Bandstra, J. W. Singer and P. Bonomi, Br. J. Cancer, 2008, 98, 1608-1613.

38 K. LBarr, T. H. Konia and M. A. Fung, J. Cutan. Pathol., 2011, 38(4), 346-350.

39 J. Conde, K. Davis, E. Nituen, N. Balmer, D. Jones and A. Mcmichael, J. Dermatol. Treat, 2010, 21(2), 122-124.

40 L. Nuhn, L. V. Hoecke, K. Deswarte, B. Schepens, Y. P. Li, B. Lambrecht, S. D. Koker, S. A. David, X. Saelens and B. D. Geest, Biomaterials, 2018, 178, 643-651.

41 F. R. Bertani, P. Mozetic, M. Fioramonti, M. Iuliani, G. Ribelli, F. Pantano, D. Santini, G. Tonini, M. Trombetta, L. Businaro, S. Selci and A. Rainer, Sci. Rep., 2017, 7(1), 8965. 42 J. Wei and G. E. Besner, J. Surg. Res., 2015, 197(1), 126-138.
43 D. P. Vasconcelos, A. C. Fonseca, M. Costa, I. F. Amaral, M. A. Barbosa, A. P. Águas and J. N. Barbosa, Biomaterials, 2013, 34, 9952-9959.

44 B. J. Ignacio, T. J. Albin, A. P. Esser-Kahn and M. Verdoes, Bioconjugate Chem., 2018, 29(3), 587-603.

45 R. Bastola, S. Lee and J. Pharm, Investigation, 2019, 49, 279-285.

46 S. Yi, S. D. Allen, Y. G. Liu, B. Z. Ouyang, X. Li, P. Augsornworawat, E. B. Thorp and E. A. Scott, ACS Nano, 2016, 10(12), 11290-11303.

47 T. K. Kishimoto and R. A. Maldonado, Front. Immunol., 2018, 9, 230.

48 Y. J. Lin, C. N. Wen, Y. Y. Lin, W. C. Hsieh, C. C. Chang, Y. H. Chen, C. H. Hsu, Y. J. Shih, C. H. Chen and C. T. Fang, Nat. Commun., 2020, 11, 315. 